\title{
A multiwavelength study of solar flare waves
}

\section{Perturbation characteristics and physical interpretation}

\author{
A. Warmuth ${ }^{1}$, B. Vršnak ${ }^{2}$, J. Magdalenić ${ }^{2}$, A. Hanslmeier ${ }^{3}$, and W. Otruba ${ }^{4}$ \\ 1 Astrophysikalisches Institut Potsdam, An der Sternwarte 16, 14482 Potsdam, Germany \\ ${ }^{2}$ Hvar Observatory, University of Zagreb, Kačićeva 26, 10000 Zagreb, Croatia \\ ${ }^{3}$ Institute for Geophysics, Astrophysics and Meteorology, University of Graz, Universitätsplatz 5, 8010 Graz, Austria \\ ${ }^{4}$ Sonnenobservatorium Kanzelhöhe, 9521 Treffen, Austria
}

Received 17 September 2003 / Accepted 26 January 2004

\begin{abstract}
The study of solar flare waves - globally propagating wave-like disturbances usually observed in $\mathrm{H} \alpha$ as Moreton waves - has recently come back into focus prompted by the observation of coronal waves in the EUV with the SOHO/EIT instrument ("EIT waves"), and in several additional wavelength channels. We study 12 flare wave events in order to determine their physical nature, using $\mathrm{H} \alpha$, EUV, helium I, SXR and radioheliographic data. In the companion Paper I, we have presented the observational data and have discussed the morphology, spatial characteristics and the kinematics of the different flare wave signatures. The wavefronts observed in the various spectral bands were found to follow kinematical curves that are closely associated, implying that they are signatures of the same physical disturbance. In the present paper, we continue the study with a close examination of the evolution of the common perturbation that causes the different wave signatures, and with a detailed analysis of the metric type II radio bursts that were associated with all flare wave events. The basic characteristics of the waves are deceleration, perturbation profile broadening, and perturbation amplitude decrease. This behavior can be interpreted in terms of a freely propagating fast-mode MHD shock formed from a large-amplitude simple wave. It is shown that this scenario can account for all observed properties of the flare waves in the various spectral bands, as well as for the associated metric type II radio bursts.
\end{abstract}

Key words. shock waves - Sun: flares - Sun: radio radiation - Sun: corona - Sun: chromosphere

\section{Introduction}

Recently, there has been a resurgence of interest in the solar flare-associated globally travelling disturbances known as Moreton or flare waves. This was prompted by the discovery of coronal waves by the EIT instrument aboard $\mathrm{SOHO}$ ("EIT waves"; Thompson et al. 1998), which may represent the coronal counterpart of the chromospheric Moreton waves (Moreton \& Ramsey 1960) that were predicted by the "sweeping-skirt" hypothesis (Uchida 1968; Uchida et al. 1973). In this scenario, a fast-mode wave or shock propagates through the corona and sweeps over the chromosphere, where it creates the Moreton wavefronts observed in $\mathrm{H} \alpha$.

Most of the recent work on the subject has been on a case study basis, and has used only a subset of the available imaging data. To obtain more conclusive results, we have conducted a multiwavelength study of flare waves using a larger data and event set.

In the previous paper of the series (Warmuth et al. 2004; henceforth Paper I) we have described morphological

Send offprint requests to: A. Warmuth,

e-mail: awarmuth@aip.de characteristics and kinematics of 12 flare waves, using imaging data from five spectral bands (H $\alpha$, EUV, helium I, SXR and microwaves) $)^{1}$. Our main result was that the flare wave signatures seen in the different spectral channels all follow closely associated kinematical curves, which implies that they are caused by a common physical disturbance. This is supported by morphological similarities. Furthermore, all flare waves are decelerating, which confirms the results obtained by Warmuth et al. (2001). Consequently, there is no "velocity discrepancy" between Moreton and EIT waves. The differences in the measured speeds can be explained by the deceleration of the disturbance, combined with the fact that its signatures can be traced to much larger distances in EIT than in $\mathrm{H} \alpha$, and the low image cadence of EIT.

In this paper we continue the study of these 12 events, focusing on the characteristics of the disturbance which causes the flare wave signatures. In order to make inferences on the physical nature of the disturbance, the evolution of its perturbation profile must be studied, which will be done in Sect. 3 .

\footnotetext{
${ }^{1}$ See Paper I for a more detailed introduction into the flare wave phenomenon.
} 
It has already been pointed out (see, e.g., Harvey et al. 1974, and references therein) that metric type II bursts are closely associated with Moreton waves, but how exactly the two phenomena are related is still not known. Therefore, we study the metric type II bursts that where present in all events (Sect. 4), and use them to make inferences on the nature of the underlying disturbance. Combined with the outcome of Paper I, the results of these studies will be employed to derive a consistent physical interpretation of flare waves (Sect. 5).

\section{Observations and techniques}

The event search and data selection methods have been discussed in Paper I (Sect. 2.1), here, we give only a brief overview. 12 suitable flare wave events were found in the interval from March 1997 to August 2001, which corresponds to the rising and the maximum phase of solar cycle 23. All events were observed in $\mathrm{H} \alpha$, using data from Kanzelhöhe Solar Observatory (KSO; Messerotti et al. 1999), Big Bear Solar Observatory (BBSO; Denker et al. 1999) and Hida Observatory (Kurokawa et al. 1995; Shibata et al. 2002). The Hida data include co-temporal filtergrams in the red and the blue wing of $\mathrm{H} \alpha( \pm 0.8 \AA)$. Additional chromospheric filtergrams (for two events) in He I were provided by the CHIP instrument (MacQueen et al. 1998) at the Mauna Loa Solar Observatory. For eight events, EUV data were available from the $\mathrm{SOHO} / \mathrm{EIT}$ instrument (Delaboudinière et al. 1995), while SXR images were provided by Yohkoh/SXT for three events (Tsuneta et al. 1991). Finally, four events were observed in the microwave range $(17 \mathrm{GHz})$ by the Nobeyama radioheliograph $(\mathrm{NoRH}$; Nakajima et al. 1994).

Radiospectrograms in the metric range were provided by the Tremsdorf radio spectral polarimeter (Mann et al. 1992) of the Astrophysical Institute Potsdam, by the Hiraiso radio spectrometer (Kondo et al. 1995), and by the Culgoora radiospectrograph (Prestage et al. 1994).

In Paper I we studied the kinematics of flare waves by derived distance-time plots $r(t)$ of the propagation of their leading edge using two different techniques: the visual determination of the leading edge of the wavefronts, where the distances of the wavefronts from a probable starting location are measured along ten paths which are parts of great circles of one solar radius, and the "profile method", where we obtain intensity profiles along a large number of paths, which are then averaged laterally over the whole sector angle, giving the mean intensity as a function of distance for each given moment, $\Delta I(r, t)$. We use the same starting point as in the visual method. From the profiles we can derive the locations of the leading edge, the intensity maximum and the trailing edge, as well as the evolution of the intensity (see Sect. 3).

The probably starting point of the waves was extrapolated by fitting a circle to the earliest $\mathrm{H} \alpha$ wavefront. The estimated starting time $t_{0 \text { est }}$ was usually taken as the time of the last $\mathrm{H} \alpha$ image without a visible wavefont. Alternative starting times have been derived using 2 nd degree polynomial $\left(t_{0 \text { poly }}\right)$ and power-law fits $\left(t_{0 \mathrm{pl}}\right)$ of $r(t)$ (see Paper I, Sect. 5.1).

In Table 1, a brief overview of all events is given (for a more detailed presentation, see Table 1 in Paper I), and the labels E1
Table 1. Event overview. Event gives the event label and date, flare imp. the optical and soft X-ray importance of the flare, 1st Moreton the time of the first Moreton wavefront, and additional waves indicates the additional spectral channels where wave signatures were observed (all events were recorded in $\mathrm{H} \alpha$ ).

\begin{tabular}{lccc}
\hline \hline Event & $\begin{array}{c}\text { Flare } \\
\text { imp. }\end{array}$ & $\begin{array}{c}\text { 1st } \\
\text { Moreton }\end{array}$ & $\begin{array}{c}\text { Additional } \\
\text { waves }\end{array}$ \\
\hline E1: 1997 Sep. 24 & 1B/M5.9 & 02:45:00 & EUV, 17 GHz \\
E2: 1997 Nov. 3 & SB/C8.6 & $04: 36: 00$ & EUV, SXR \\
E3: 1997 Nov. 3 & 1B/M1.4 & $09: 09: 10$ & EUV, SXR \\
E4: 1997 Nov. 4 & 2B/X2.1 & $05: 58: 00$ & EUV \\
E5: 1998 May 2 & 3B/X1.1 & $13: 38: 57$ & EUV \\
E6: 1998 Aug. 8 & 1B/M3.0 & $03: 16: 00$ & 17 GHz \\
E7: 1998 Aug. 18 & 1B/X4.9 & $22: 17: 57$ & - \\
E8: 1998 Aug. 19 & 2B/X3.9 & $21: 47: 54$ & - \\
E9: 1998 Aug. 24 & 3B/X1.0 & $22: 03: 11$ & He I \\
E10: 2000 Mar. 2 & SN/M6.5 & $13: 39: 24$ & EUV \\
E11: 2000 Mar. 3 & 1B/M3.8 & $02: 12: 00$ & EUV, 17 GHz \\
E12: 2000 Nov. 25 & 2B/X1.9 & $18: 39: 10$ & EUV, He I \\
\hline
\end{tabular}

to E12 are associated with each flare wave event. Figure 1 in Paper I presents the Moreton and EIT wavefronts for all events, including the sectors in which the kinematics and profiles of the wavefronts were measured.

\section{Perturbation profile evolution}

\section{1. $H \alpha$}

For all events, intensity profiles $\Delta I(r, t)$ of the $\mathrm{H} \alpha$ perturbations were obtained according to the scheme described in Sect. 2. From these profiles the maximum of the relative intensity, $I_{\mathrm{m}}(t)$, and the locations of the leading edge, $r_{\mathrm{b}}(t)$, the intensity maximum, $r_{\mathrm{m}}(t)$, and the trailing edge, $r_{\mathrm{e}}(t)$, were determined. The perturbation thickness was defined as $\Delta r(t)=r_{\mathrm{b}}(t)-r_{\mathrm{e}}(t)$. As an example, Fig. 1 shows the evolution of these parameters for all events that were observed only in the $\mathrm{H} \alpha$ line center. For the same events, the original $\mathrm{H} \alpha$ line center intensity profiles are presented in Fig. 2. For $r$ and $\Delta r$ in Fig. 1, the error bars represent the estimated measurement errors that result from the lack of sharp edges (particularly for $r_{\mathrm{e}}$ ). The error bars for $I_{\mathrm{m}}$ in Fig. 1 and for all profiles in Figs. 2 and 3 show the standard deviation of the intensity fluctuations that are obtained by calculating the ratio of the consecutive profiles (measuring only the quiet parts of the profile, i.e. ahead of the wavefronts).

In all events, the plots of $r_{\mathrm{b}}(t)$ show deceleration, which has already been found with the "visual" method (see Paper I, Sect. 5.1). In most events, the wavefront thickness $\Delta r(t)$ is increasing. Only in E4, $\Delta r(t)$ was decreasing in the $\mathrm{H} \alpha$ line center and remained rather constant in the wings, while in E9 (Fig. 1b), $\Delta r(t)$ remained constant in the line center. This deviant behavior may be, at least partially, a consequence of the difficulty of determining the leading and trailing edges from the profiles. This is why we preferred to use the "visual" values 


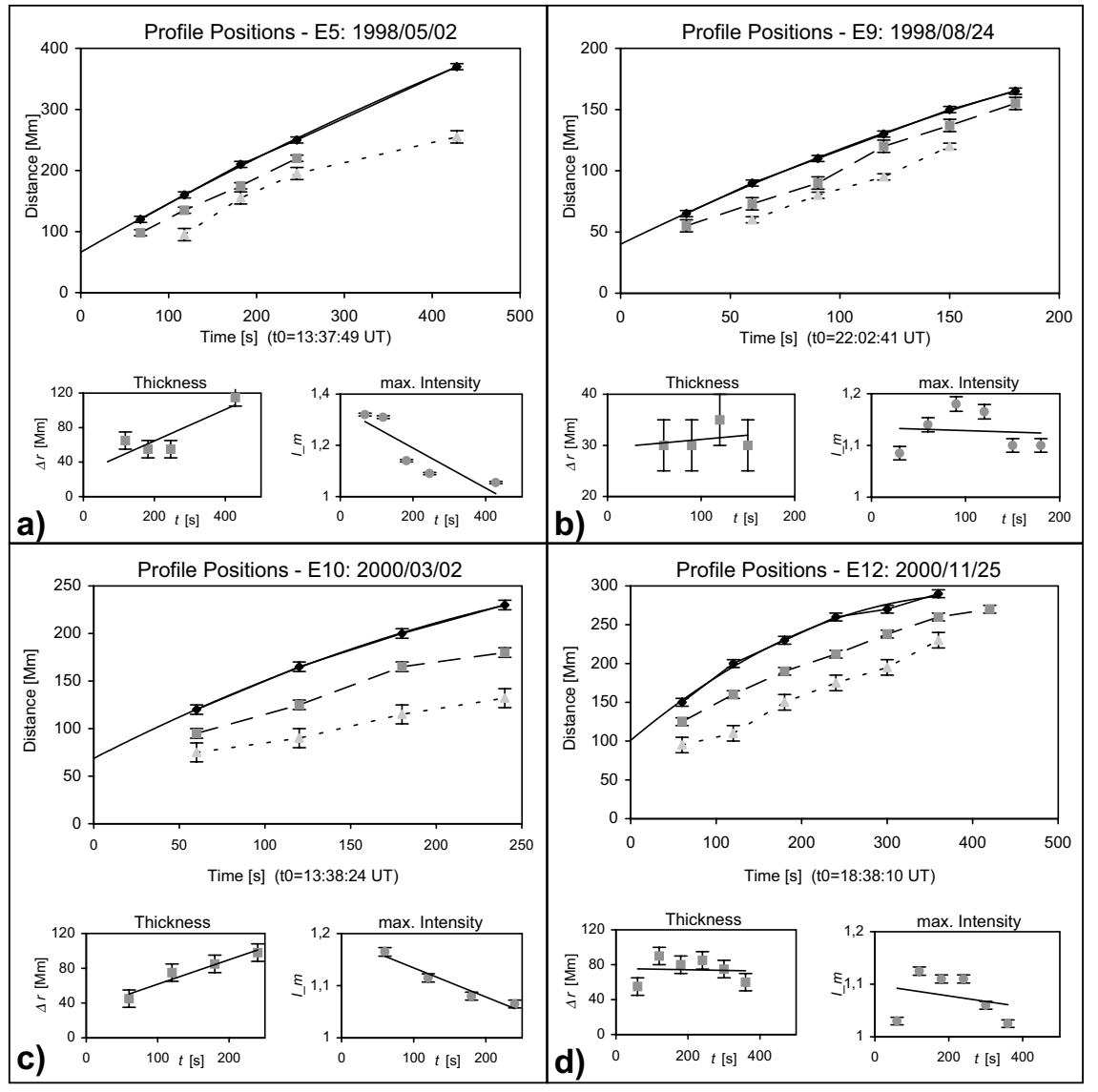

Fig. 1. The kinematics of the leading edges $r_{\mathrm{b}}$ (diamonds), the intensity maximum $r_{\mathrm{m}}$ (squares), and the trailing edges $r_{\mathrm{e}}$ (triangles) of the Moreton waves of a) E5, b) E9, c) E10, and d) E12. For $r_{\mathrm{b}}$, a 2nd degree polynomial least-squares fit is shown. The smaller graphs on the left below the main plots show the development of the wavefront thickness $\Delta r$ (in $\mathrm{Mm}$ ), while those on the right show the evolution of the maximum intensity $I_{\mathrm{m}}$ (in arbitrary units). Linear fits are shown for $\Delta r$ and $I_{\mathrm{m}}$. for the discussion of the kinematics in Paper I. However, on average we found a good agreement between the kinematical parameters of the leading edges derived from the "visual" and the "profile" method (see Paper I, Table 3).

The evolution of the maximum intensity $I_{\mathrm{m}}(t)$ shows an even clearer trend: in all events $I_{\mathrm{m}}(t)$ decreases with increasing time/distance. Of the seven Moreton waves where $I_{\mathrm{m}}(t)$ was determined from the line center data, two show a monotonous decrease in intensity, while five display an initial rise (see Figs. 1b and 1d) - usually between the first two observed wavefronts and a subsequent monotonous decrease.

\section{2. $\mathrm{H} \alpha$ line wings}

In E2, E4, E6 and E11, intensity profiles were obtained in the center of the $\mathrm{H} \alpha$ line (henceforth $\mathrm{H} \alpha_{\mathrm{c}}$ ), as well as in the red and in the blue line wing ( $\mathrm{H} \alpha_{r}$ and $\mathrm{H} \alpha_{\mathrm{b}}$, respectively). In $\mathrm{H} \alpha_{r}$, the wave is observed as an absorption front, which is followed by an emission front. The situation for $\mathrm{H} \alpha_{\mathrm{b}}$ is vice versa. This reproduces the results from the morphological study (Paper I, Sect. 3.1).

Generally, the dark wave seen in $\mathrm{H} \alpha_{r}$ tends to lead the other fronts slightly, the thickness $\Delta r$ is roughly similar in all three bands, and broadening and intensity decrease are also observed in the wings. Some profiles show evidence of a third front behind the two wavefronts discussed above. This additional front has the same sense of $\Delta I$ as the leading front, and could be interpreted as a slight downward movement of the chromosphere after overshooting its equilibrium height during the upward expansion which causes the first trailing front.

The behavior of the disturbances becomes somewhat clearer when the profiles from $\mathrm{H} \alpha_{\mathrm{b}}$ are combined with those from $\mathrm{H} \alpha_{r}$ to derive a Doppler signal profile

$v_{\mathrm{D}}(r)=\frac{\Delta I_{\mathrm{H} \alpha_{r}}(r)-\Delta I_{\mathrm{H} \alpha_{\mathrm{b}}}(r)}{\Delta I_{\mathrm{H} \alpha_{r}}(r)+\Delta I_{\mathrm{H} \alpha_{\mathrm{b}}}(r)}$.

$v_{\mathrm{D}}$ gives a crude measure for the Doppler velocity of the chromosphere in arbitrary units. It assumes that the only influence on the $\mathrm{H} \alpha$ line is a Doppler shift, i.e. it neglects changes in the line profile which may be present due to a temperature/pressure increase.

The Doppler profiles $v_{\mathrm{D}}(r, t)$ are shown in Fig. 3 for all four events with line wing data coverage. All profiles show an initial downward motion $\left(v_{\mathrm{D}}<0\right)$ and a subsequent upward swing. The Doppler profiles reveal that the downward motion is more impulsive than the upward swing, i.e. it has a larger amplitude $v_{\mathrm{D}}(r)$ and a larger velocity gradient $\partial v_{\mathrm{D}}(r) / \partial r$.

\subsection{Helium I}

Perturbation profiles obtained in He I show that the He I disturbance actually has a two-step shape: a shallow frontal segment and a more pronounced main perturbation element. The comparison of co-temporal $\mathrm{He} \mathrm{I}$ and $\mathrm{H} \alpha$ profiles performed by Vršnak et al. (2002a) reveals that the beginning of the second (large) step corresponds to the beginning of the $\mathrm{H} \alpha$ 

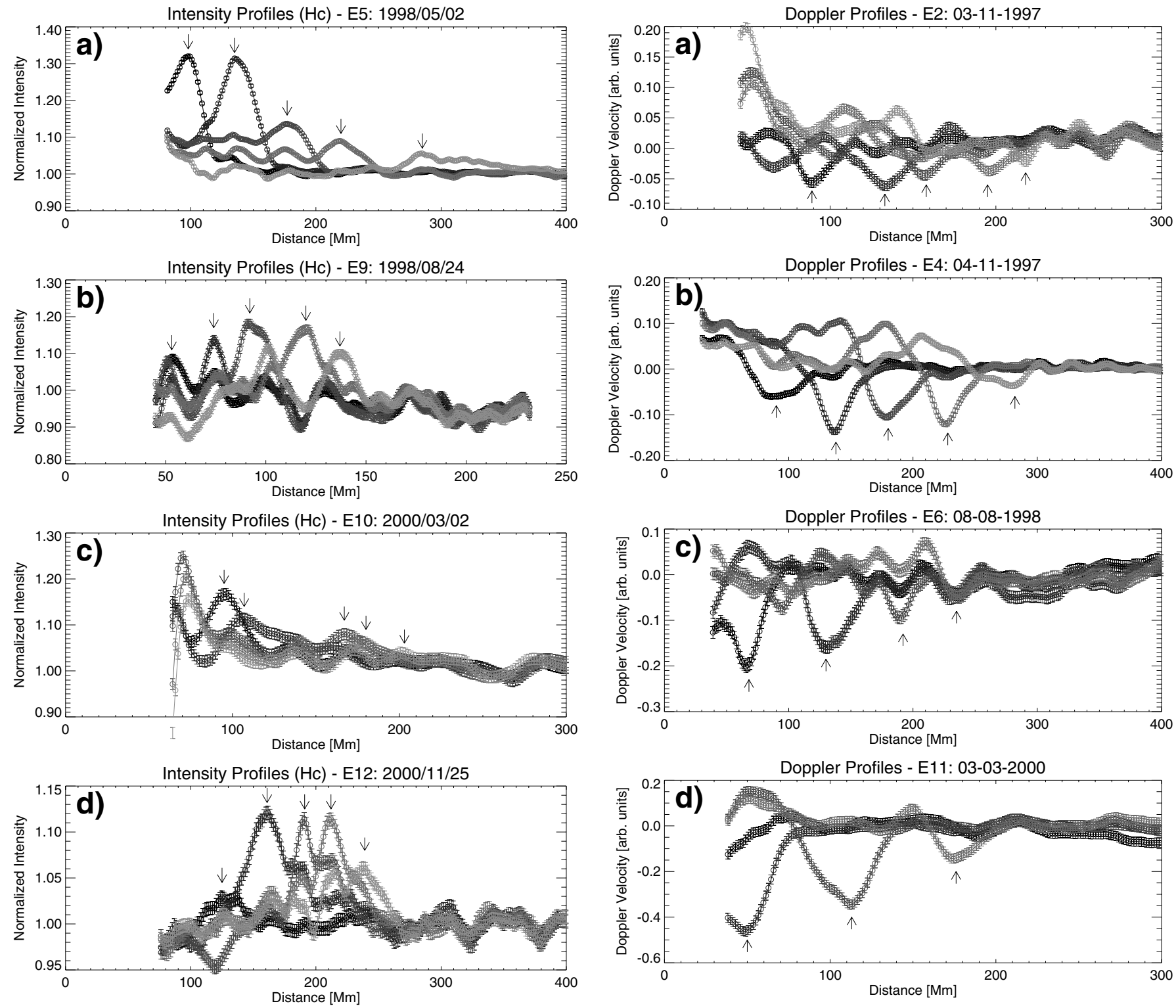

Fig. 2. The $\mathrm{H} \alpha$ line center intensity profiles $\Delta I_{\mathrm{c}}(r, t)$ for a) $\mathrm{E} 5$, b) E9, c) E10, and d) E12. Only the first five wavefronts are included. With increasing time, the profiles are plotted in lighter shades of gray. The location of the maximum of the perturbation $\left(I_{\mathrm{m}}\right)$ is indicated by arrows for all profiles.

perturbation, while the frontal segment (the "forerunner") leads the $\mathrm{H} \alpha$ front by $\approx 30 \mathrm{Mm}$. This is consistent with the results given by the "visual" method (see Sect. 5.3 and Fig. 9f in Paper I).

\subsection{EIT, SXT and NoRH}

Due to the low image cadence of EIT (in all events but E1 only two fronts were recorded), it is not possible to study the evolution of the perturbation profile of EIT waves in the same manner as it was done for $\mathrm{H} \alpha$ and $\mathrm{He}$. What makes matters worse is that the "second" EIT fronts (the ones at larger $r$ ) are sometimes not seen in the profiles because they are too diffuse and/or fragmented. However, the data are sufficient to obtain some general results. For the EIT fronts that are near

Fig. 3. The Doppler velocity profiles $v_{\mathrm{D}}(r, t)$ for a) E2, b) E4, c) E6, and d) E11. With increasing time, the profiles are plotted in lighter shades of gray. The location of the maximum downward velocity is indicated by arrows.

the origin (E1, E3, and E5; with $r<300 \mathrm{Mm}$ ), peak intensities of $I_{\mathrm{m}}=1.4-2.1$ times the undisturbed background intensity were measured, while $I_{\mathrm{m}}$ amounted to only $1.05-1.3$ for the seven wavefronts that could be measured at distances of $r=400-900 \mathrm{Mm}$. As for the thickness $\Delta r$, the three "close" fronts amounted to $\Delta r=40-70 \mathrm{Mm}$, while the seven "far" ones had significantly larger thicknesses, ranging from $150 \mathrm{Mm}$ to $320 \mathrm{Mm}$. Once again, these results are consistent with the morphological studies (see Paper I, Sect. 3.2) and confirm the perturbation amplitude decrease and the thickness increase as general properties of flare waves.

The SXR wave of E2 was too faint to yield meaningful profiles, while in E3 only two fronts were observed, and there are problems with saturation and scattered light from the flare. We therefore refrained from studying the profiles of the 
Table 2. Associated metric type II bursts. $t_{\mathrm{b}}$ is the starting time of the type II burst, $f_{\mathrm{b}}$ the starting frequency (in MHz), $r_{\mathrm{b}}$ the inferred starting height above the solar surface (in $\mathrm{Mm}$ ), $v_{\mathrm{b}}$ and $\bar{v}$ the initial and the average speed, respectively (in $\mathrm{km} \mathrm{s}^{-1}$ ), $\bar{a}$ the average deceleration (in $\mathrm{m} \mathrm{s}^{-2}$ ), and $B D W_{0}$ and $\overline{B D W}$ are the initial and the average bandsplit, respectively. Values in brackets are ambiguous, and a blank space indicates that the parameter could not be measured at all.

\begin{tabular}{|c|c|c|c|c|c|c|c|c|}
\hline Event & $t_{\mathrm{b}}$ & $f_{\mathrm{b}}$ & $r_{\mathrm{b}}$ & $v_{\mathrm{b}}$ & $\bar{v}$ & $\bar{a}$ & $B D W_{0}$ & $\overline{B D W}$ \\
\hline E1 & $02: 47: 50$ & (73) & (369) & (1200) & & & $(0.3)$ & \\
\hline E2 & 04:38:06 & 85 & 323 & 1009 & 769 & -2634 & 0.53 & 0.49 \\
\hline E3 & 09:08:50 & 143 & 179 & 1535 & 1038 & -1113 & 0.51 & 0.46 \\
\hline E4 & 06:01:12 & 105 & 252 & 1686 & 1208 & -3015 & 0.60 & 0.40 \\
\hline E5 & $13: 40: 50$ & (166) & (143) & $(530)$ & (277) & $(-812)$ & & \\
\hline E6 & 03:17:00 & 192 & 114 & 2629 & 1127 & -3593 & & \\
\hline E7 & $22: 16: 00$ & 244 & 77 & & & & 0.43 & \\
\hline E8 & 21:45:00 & 100 & 258 & 530 & & & & \\
\hline E9 & 22:03:00 & (75) & (397) & & & & $(0.33)$ & \\
\hline E10 & $13: 41: 00$ & (29) & (793) & (1655) & (1637) & $(-6216)$ & $(0.30)$ & $(0.31)$ \\
\hline E11 & 02:13:06 & 259 & 66 & 1649 & 1366 & -2592 & & \\
\hline E12 & $(18: 39: 00)$ & & & & & & & \\
\hline Mean: & & 161 & 181 & 1506 & 1101 & -2589 & 0.52 & 0.45 \\
\hline$\sigma:$ & & 71 & 100 & 709 & 222 & 918 & 0.07 & 0.04 \\
\hline
\end{tabular}

SXR waves. The NoRH radioheliograms, on the other hand, are much too noisy (due to image reconstruction artefacts) to determine meaningful profiles.

\section{Associated metric type II radio bursts}

11 events in our sample were accompanied by metric type II radio bursts, and in the remaining event, E5, three emission features were classified as type II-like bursts (Pohjolainen et al. 2001) based on their drift rate and fine structure. This indicates that Moreton waves are generally associated with a shock propagating outward through the corona. In Table 2 the basic type II burst parameters are shown. Note that in many events several parameters could not be determined, or at least not with sufficient accuracy (the values in brackets in Table 2). These inaccurate parameters will not be used for deriving mean values or for correlation studies (Sect. 5.5). The kinematical parameters were determined using the 5-fold Saito density model (Saito 1970), which is appropriate for the active region (henceforth AR) corona. For details on the measurement method, see Vršnak et al. (2001).

The average source velocity inferred from the burst drift rates is $\left\langle\bar{v}_{\mathrm{II}}\right\rangle=1101 \pm 222 \mathrm{~km} \mathrm{~s}^{-1}$, which is significantly higher than the typical type II velocities (e.g. Robinson et al. 1985 found $\left\langle\overline{\mathrm{I}}_{\mathrm{II}}\right\rangle=764 \pm 65 \mathrm{~km} \mathrm{~s}^{-1}$ ) and also higher than the average velocity of type II bursts that are associated with EIT waves, given by Klassen et al. (2000) as $739 \mathrm{~km} \mathrm{~s}^{-1}$. The inferred source speeds are also higher than the velocities of the associated flare waves (e.g., the initial $\mathrm{H} \alpha$ wave velocity derived from the first observed wavefront pair was $\left\langle v_{1}\right\rangle=845 \pm 162 \mathrm{~km} \mathrm{~s}^{-1}$ ).

In all bursts, the velocities evaluated by using the Saito density model show a deceleration $\left(\left\langle\bar{a}_{\mathrm{II}}\right\rangle=-2589 \pm 918 \mathrm{~m} \mathrm{~s}^{-2}\right)$, which is on average about twice as large as for the Moreton waves. This need not necessarily mean that they are really decelerating more strongly than the Moreton waves, since the kinematical parameters of type II bursts must generally be regarded with caution as they are inherently dependent on the coronal density model that is used for converting the observed frequencies to geometrical heights. It may be that the density above a complex AR is not reliably represented by a simple radial density model, or that the source motion is strongly inclined to the vertical (Klassen et al. 1999; Klein et al. 1999). This could introduce an artificial, or artificially large, deceleration.

The mean of the starting frequencies of the fundamental band emission was $\left\langle f_{\mathrm{b}}\right\rangle=161 \pm 71 \mathrm{MHz}$ (corresponding to a height of $\approx 200 \mathrm{Mm}$ ), which is considerably higher in frequency than the values found for an accidental type II burst sample (around $80 \mathrm{MHz}$; Vršnak et al. 2001). This is consistent with the results obtained by Vršnak \& Lulić (2000a) who deduced that faster bursts should be formed at lower heights.

Four of the events showed a well defined band-split of the emission lanes (Nelson \& Melrose 1985). The relative bandsplit is defined as $B D W=\left(f_{\mathrm{h}}-f_{1}\right) / f_{1}$, where $f_{\mathrm{h}}$ and $f_{1}$ are the higher and lower emission frequencies of the split band (Vršnak et al. 2002b). The average initial band-split amounted to $\left\langle B D W_{0}\right\rangle=0.52$, and the average value of the mean bandsplit was $\langle\overline{B D W}\rangle=0.45$. These values are considerably larger than usual ones ranging from 0.15 to 0.35 (Vršnak et al. 2002b).

Interpreting the band-split in terms of plasma emission from the upstream and downstream shock region (Vršnak et al. 2001), $B D W$ can be associated with the density jump $X=\varrho_{\mathrm{d}} / \varrho_{\mathrm{u}}$ at the shock front $\varrho_{\mathrm{d}}$ and $\varrho_{\mathrm{u}}$ are the densities down- and upstream of the shock, respectively). Since the plasma 
frequency is related to the electron density as $f \propto \sqrt{n}$, one finds $X=(1+B D W)^{2}$ (Vršnak et al. 2001). The values $B D W=0.52$ and 0.45 then correspond to $X=2.3$ and 2.1, respectively.

The density jump $X$ can be used to estimate the Alfvénic Mach number $M_{\mathrm{A}}$ of the shock (e.g., Vršnak et al. 2002b), which is defined by the ratio of the speed of the disturbance to the Alfvén speed $v_{\mathrm{A}}$. In the case of perpendicular propagation the following relation holds:

$M_{\mathrm{A}}=\sqrt{\frac{(5 \beta+5+X) X}{2(4-X)}}$,

where $\beta$ is the plasma-to-magnetic pressure ratio. Substituting $X=2.3$ and 2.1 into Eq. (2) one finds $M_{\mathrm{A}}=2.22$ and 1.98 for $\beta=0$, and $M_{\mathrm{A}}=2.88$ and 2.59 for $\beta=1$, respectively. Comparing this with the values $M_{\mathrm{A}}(\beta=0)=1.2-1.6$ usually found in type II bursts (Vršnak et al. 2002b), it is again evident that Moreton-associated type II bursts are more energetic than the average.

Fast-mode waves propagating perpendicularly to the magnetic field lines $(v \perp B)$ travel at the magnetosonic speed

$v_{\mathrm{ms}}=\left(v_{\mathrm{A}}^{2}+c_{\mathrm{s}}^{2}\right)^{1 / 2}$,

where $c_{\mathrm{s}}$ is the sound speed $\left(c_{\mathrm{s}}^{2}=\gamma p / \varrho\right.$, where $p$ and $\varrho$ are the gas pressure and the mass density, respectively). The magnetosonic Mach number can then be evaluated utilizing $v_{\mathrm{ms}}=v_{\mathrm{A}}\left(1+c_{\mathrm{s}}^{2} / v_{\mathrm{A}}^{2}\right)^{1 / 2}=v_{\mathrm{A}}(1+5 \beta / 6)^{1 / 2}$, where we have substituted for the sound-to-Alfvén speed ratio $\left(c_{\mathrm{s}} / v_{\mathrm{A}}\right)^{2}=\gamma \beta / 2$, with the ratio of specific heats $\gamma=5 / 3$. So, one finds $M_{\mathrm{ms}}=$ $M_{\mathrm{A}}(1+5 \beta / 6)^{-1 / 2}$. For $\beta=1$, the obtained values of $M_{\mathrm{A}}$ for the type II bursts correspond to magnetosonic Mach numbers ranging between $M_{\mathrm{ms}}=2.13$ and 1.91 (for $\beta=0, M_{\mathrm{ms}}=M_{\mathrm{A}}$ ).

In E3, the burst source could be localized in decimetricmetric radioheliograms (Khan \& Aurass 2002) from the Nançay Radioheliogaph (NRH; Kerdraon \& Delouis 1997). The locations of the burst sources at the different NRH frequencies at the times of the Moreton, EIT and SXT wavefronts suggest a close association of the burst with the flare wave. In E5, three fast-moving sources $\left(v=800-890 \mathrm{~km} \mathrm{~s}^{-1}\right)$ in the $\mathrm{NRH}$ radioheliograms could be identified with the type II-like bursts (Pohjolainen et al. 2001). They appear at locations in close temporal and spatial coincidence with the extrapolated location of the Moreton wave. Pohjolainen et al. (2001) suggested that these bursts could result from the interaction of the wave with pre-existing coronal structures. The fact that two of the sources coincided with bright regions seen in SXT and EIT images gives some support to this scenario.

\section{Summary and interpretation}

\subsection{Association and kinematics}

Signatures of flare waves were found in five different spectral bands - H $\alpha$ (line center and wings), EUV, He I, SXR and $17 \mathrm{GHz}$. It seems that a disturbance of sufficient strength will generate signatures in all of these spectral bands (cf. Table 1 in Paper I). The waves were found to be following closely associated kinematical curves (Paper I, Sect. 5), which suggests that they are caused by the same physical disturbance. In the case of $\mathrm{H} \alpha$ and EIT waves, the close association is directly evident from the observations of nearly cospatial (and morphologically similar) $\mathrm{H} \alpha$ and EIT wavefronts in E1 and especially in E5 (see Paper I, Sect. 3.2). Conversely to what is reported by Eto et al. (2002) and Harra \& Sterling (2003), and to what is predicted by the model proposed by Chen et al. (2002), we did not find evidence for the presence of two separate waves.

Furthermore, it was found that all flare waves are decelerating (Paper I, Sect. 5). This causes an artificial velocity discrepancy between EIT and $\mathrm{H} \alpha$ signatures: the EIT signatures must show lower mean velocities than their $\mathrm{H} \alpha$ counterparts because they can be traced to much larger distances, where they have already significantly decelerated.

\subsection{Perturbation characteristics and evolution}

An important finding is that deceleration is a general characteristic of flare waves, regardless of the spectral band in which they were observed. Consequently also the underlying physical disturbance must be decelerating. The deceleration is not constant, it becomes smaller with increasing time and distance.

The analysis of the intensity profiles $\Delta I(r, t)$ of the flare waves has revealed two main results: the broadening of the wavefronts $(\partial / \partial t \Delta r(t)>0)$ and the decrease of the maximum intensity $\left(\partial / \partial t I_{\mathrm{m}}(t)<0\right)$. More evidence of broadening is given by the fact that sharp EIT waves ( $\mathrm{S}$-waves) are only observed close to the presumed source location (e.g. in E1 and E5), while the EIT fronts that are observed farther away are always broader and more diffuse.

In all events, the maximum intensity $I_{\mathrm{m}}$ of the wavefronts was either monotonically decreasing, or showed a short initial rise (only during the early propagation, usually between the first two wavefronts). The decrease of the maximum intensity can be interpreted in terms of the decline of the amplitude of the perturbation. On the other hand, the disturbance may still increase in amplitude early on. It is reasonable to assume that the amplitude and steepness of the leading part of the perturbation have to exceed a certain threshold in order to generate an observable $\mathrm{H} \alpha$ signature. The rather large initial distances of the earliest observed Moreton fronts $\left(\left\langle r_{0}\right\rangle=49 \pm 30 \mathrm{Mm}\right.$; see Paper I, Sect. 5.1) can therefore be interpreted as the result of the period needed to form a segment of the perturbation that is steep enough to affect the chromospheric plasma.

\subsection{The fast-mode MHD wave}

The morphological studies have shown that Moreton fronts are an enhancement of pre-existing chromospheric structures (Paper I, Sect. 3.1). Their leading edges agree nicely with a circular fit over a rather large distance range (Sect. 4.1 in Paper I), and the calculation of Doppler signal profiles (Sect. 3.2) has revealed the down-up swing of the chromosphere. These results indicate that the traveling disturbance is a wave, and not, for example, propagating ejected matter. It is also difficult to see how a wavefront with a nearly perfect circular curvature 

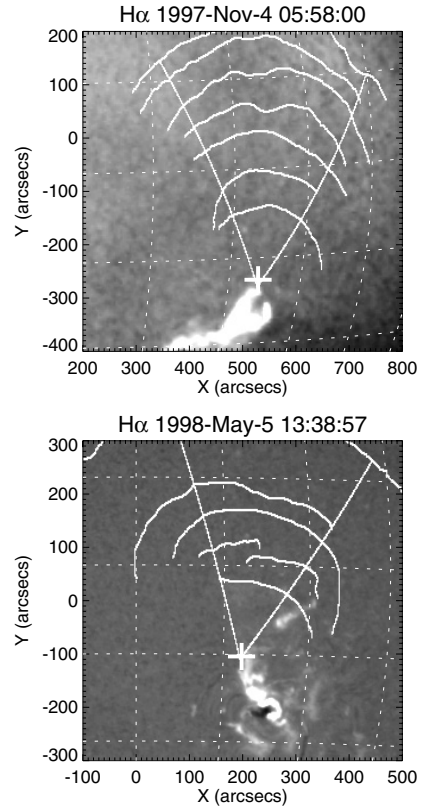

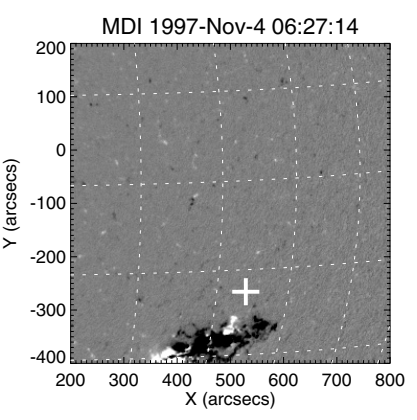

MDI 1998-May-5 12:48:04

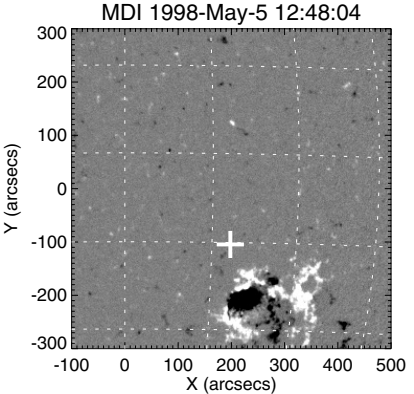

SXT 1997-Nov-4 05:49:48

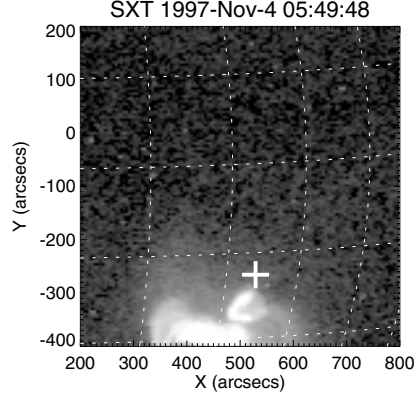

SXT 1998-May-5 13:31:14

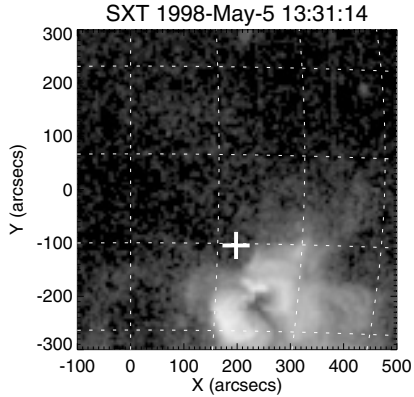

EIT 195 1997-Nov-4 05:40:01
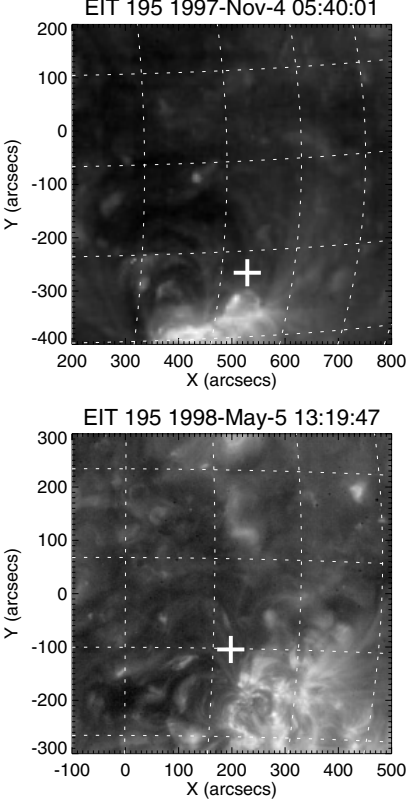

Fig. 4. The vicinity of the active regions associated with E4 (NOAA 8100; top row) and E5 (NOAA 8210; bottom row). Included are (from left to right) $\mathrm{H} \alpha$ images (E4: Hida Observatory; E5: KSO), SOHO/MDI magnetograms, Yohkoh/SXT (taken with the Al.1 filter, corresponding to a peak transmission at $\approx 9 \AA)$ and $S O H O / E I T(195 \AA)$ images. The magnetograms are linearly scaled between $\pm 200 \mathrm{G}$, while the SXT and EIT frames are logarithmically scaled. On all images, the extrapolated wave source location is shown as a white cross, while the H $\alpha$ images are also overplotted with the Moreton wavefronts and the sectors in which the measurements were carried out.

could be generated by a mechanism like the one suggested by Delannée (2000), where the expanding magnetic field lines in a CME event interact with the surrounding field lines to produce compression of the plasma. Such a mechanism may actually be able to generate the more common irregular EIT fronts, but not the smooth $\mathrm{S}$-waves, let alone the Moreton wavefronts.

In a magnetized plasma there are three MHD wave modes (e.g., Priest 1982). The slow and the Alfvénic mode can be excluded from our discussion since neither can travel perpendicular to the magnetic field lines, which flare waves obviously do without difficulty. Therefore, the fast-mode MHD wave is the most probable candidate for the physical disturbance responsible for flare waves. Since flare waves propagate predominantly perpendicular to the magnetic field lines, the perturbation can be treated as a magnetosonic wave, which propagates at the velocity of $v_{\mathrm{ms}}$ (Eq. (3)).

\subsection{The fast-mode MHD shock}

Several numerical simulations of EIT waves have been carried out showing that the assumption of a fast-mode MHD wave as the cause of EIT waves is reasonable (Wang 2000; Wu et al. 2001; Ofman \& Thompson 2002). Wang concluded that EIT waves are caused by fast-mode MHD waves, and suggested that Moreton waves (and type II bursts), on the other hand, may be caused by a rather strong super-magnetosonic shock. The shock that propagates upwards would be observed as a type II burst, while the part of the shock that propagates horizontally would be slowed down by the denser plasma, finally decaying to an ordinary fast-mode wave. The Moreton wave could then be associated with the shock, while the
EIT wave would evolve from a shock to a weak fast-mode disturbance.

To establish the shock nature of Moreton waves, their characteristic speeds must be compared with the magnetosonic speed, $v_{\mathrm{ms}}$. In several recent studies (Mann et al. 1999; Wang 2000; Wu et al. 2001), magnetosonic speeds of a few $100 \mathrm{~km} \mathrm{~s}^{-1}$ were inferred for the low corona in the quiet sun, while Narukage et al. (2002), using SXT observations, obtained somewhat higher values of $v_{\mathrm{ms}}=350-600 \mathrm{~km} \mathrm{~s}^{-1}$. It could be objected that the Moreton waves, which initially propagate near an AR, encounter a medium with a much higher $v_{\mathrm{ms}}$, and that they are therefore not initially shocked. This scenario would also explain the observed deceleration. However, in all events the flares lay in the outskirts of the ARs, and the calculated wave sources were located at the edges of the flares (cf. Paper I, Fig. 1 and Sect. 4.3).

As an example, Fig. 4 shows the vicinity of the ARs that were associated with E4 and E5, respectively, in $\mathrm{H} \alpha$ images, SOHO/MDI (Scherrer et al. 1995) magnetograms, Yohkoh/SXT and $\mathrm{SOHO} / \mathrm{EIT}$ images. Also shown are the Moreton wavefronts and the extrapolated wave source locations. The magnetograms show that the Moreton waves traverse only quiet regions (with an average field strength of only a few Gauss). In addition, the SXR and EUV images prove that the observed propagation of the waves takes place well away from the AR-associated density/temperature enhancement. This is true for all events. The only region where $v_{\mathrm{ms}}$ might be enhanced is the surrounding of the source region itself, but note that our wave speed measurements rely exclusively on the observed wavefront pairs, which are invariably well removed from the source. If we take a typical magnetic field strength 
of $B=5 \mathrm{G}$, an electron number density at the coronal base of $N_{\mathrm{e}}=8.78 \times 10^{8} \mathrm{~cm}^{-3}$ as given by a one-fold Newkirk model (Newkirk 1961), which is generally accepted to be a good fit for quiet coronal conditions, and a coronal temperature of $1.4 \mathrm{MK}$, we obtain $v_{\mathrm{ms}}=387 \mathrm{~km} \mathrm{~s}^{-1}$, which is in agreement with the values cited above.

It can thus be concluded that the disturbances causing the Moreton waves are initially supermagnetosonic in all cases. For instance, the initial $\mathrm{H} \alpha$ wave speed derived from the earliest observed wavefront pairs is $\left\langle v_{1}\right\rangle=845 \pm 162 \mathrm{~km} \mathrm{~s}^{-1}$ (see Table 3 in Paper I). Taking that the magnetosonic speed in the low corona is probably in the range between 200 and $600 \mathrm{~km} \mathrm{~s}^{-1}$, this yields a mean initial magnetosonic Mach number of $\left\langle M_{\mathrm{ms}}\right\rangle=1.4-4.2$.

These rather high Mach numbers imply that the perturbation may initially be a relatively strong shock. The velocities at large distances as given by the EIT waves (with a mean velocity of $\left\langle\bar{v}_{\mathrm{EIT}}\right\rangle=311 \pm 84 \mathrm{~km} \mathrm{~s}^{-1}$ ), on the other hand, are fairly consistent with the presumed values of $v_{\mathrm{ms}}$. Therefore, the observed velocities support the assumption that flare waves (at least those which are associated with $\mathrm{H} \alpha$ Moreton waves) are generated by initially strong shocks which gradually decay to ordinary fast-mode waves $^{2}$.

The basic features of the observed disturbances - deceleration, profile broadening and decrease of amplitude - are evolutionary characteristics expected for shock waves that are formed from a large amplitude simple wave (Landau \& Lifshitz 1987; Mann 1995). The signs of decay and attenuation displayed by all waves are typical for such freely propagating shock waves, in contrast to shocks directly driven by a piston which provides a permanent energy supply. Although the evolution of a piston-driven shock strongly depends on the kinematics of the piston, it is reasonable to assume that a driven shock would show less signs of decay over a significant part of its propagation.

As the shocked perturbation propagates, the profile broadens because the leading edge moves faster than the trailing one (Landau \& Lifshitz 1987). The front edge propagates at the supermagnetosonic velocity $v_{\mathrm{b}}=M_{\mathrm{ms}} v_{\mathrm{ms}}$ whereas the trailing one propagates at $v_{\mathrm{e}}=v_{\mathrm{ms}}$ (neglecting the rarefaction segment). It should be noted that the measured $v_{\mathrm{e}}$ was not always constant: most often it displayed slight deceleration, which implies that the real trailing edge was in fact not resolved.

As the shock propagates, the dissipation of energy at the shock, the broadening of the profile, and the expansion of the shock front cause the decrease of the amplitude of the perturbation, and consequently its velocity and Mach number. This also explains why the deceleration rate decreases with increasing time and distance: as the shock slows down, the dissipation as well as the rate of broadening will decrease, and consequently the deceleration will become progressively smaller. The shock may eventually decay to an ordinary fast-mode wave with $v=v_{\mathrm{ms}}\left(M_{\mathrm{ms}}=1\right)$.

\footnotetext{
2 This scenario was already proposed by Warmuth et al. (2001), but due to the limited data set (two wave events observed in $\mathrm{H} \alpha$ and with EIT), the conclusion was less well established than in the present study.
}

Under ideal conditions the small-amplitude wave should travel at a constant velocity (provided $v_{\mathrm{ms}}$ is constant). However, it is quite probable that due to the fact that the corona is highly structured at small spatial scales, dissipation cannot be neglected even for ordinary fast-mode waves, which would still decelerate, though at a smaller rate than the shock does (see Murawski et al. 2001).

As shown above, a shock with a larger amplitude is faster and suffers a higher rate of deceleration. This notion is supported by the observation that faster flare waves tend to display larger deceleration coefficients. For various combinations of velocities (derived from both the $\mathrm{H} \alpha$ and the combined data) with the deceleration parameters $\left(\bar{a}_{\mathrm{H} \alpha}\right.$ and $\left.\bar{a}_{c b}\right)$, correlations were found, with correlation coefficients ranging up to $C=0.9$. In Figs. 5a-d, some examples are shown $\left(v_{0 \text { poly }}\right.$ refers to the speed given by the polynomial fits of $r(t)$ at $r=0)$. It is surprising that $a_{\mathrm{H} \alpha}$ versus $v_{1}$ shows the weakest trend. This is probably due to the fact that $v_{1}$ is derived from only two wavefronts, which means that the measurement errors will be larger than for velocities derived from the fits, where many more wavefronts are involved and the errors tend to be smoothed out. Moreover, due to the small number of data points (depending on the available combination of parameters) all these correlations must certainly be regarded with caution. Nevertheless, they do show the general tendency that faster waves are also decelerating more strongly, which supports the interpretation of flare waves as signatures of a decaying shock.

Another line of evidence for the presence of a shock formed from a large-amplitude simple wave is given by the time and distance of the earliest observed $\mathrm{H} \alpha$ wavefronts, provided that the waves become observable roughly at the time of the completion of the shock (this is supported by the observation that Moreton waves are only visible for $M_{\mathrm{ms}}$ larger than, say, 1.5). Theory predicts that a shock formed from a perturbation with a larger amplitude will start to form earlier and will also be completed quicker than a less energetic shock (Vršnak \& Lulić 2000a). We define a time delay between the probable initiation of the disturbance and the time at which the first wavefront is observed $\left(t_{1 M}\right)$ as $\Delta t=t_{1 M}-t_{0}$. In Figs. 5e and 5f we have plotted $\Delta t$, using $t_{0 \text { poly }}$ and $t_{0 \mathrm{pl}}$ ) as $t_{0}$, respectively, against the initial $\mathrm{H} \alpha$ velocity, $v_{1}$. Indeed, we find that the first wavefronts become visible earlier when the waves had higher velocities $(C=0.59$ and 0.74$)$.

EIT waves can still be detected at low Mach numbers, whereas the $\mathrm{H} \alpha$ disturbances are visible only in earlier stages when the Mach number is still relatively high (presumably because it is more difficult to perturb the inert chromosphere). This could explain the higher rate of occurrence of EIT waves compared to Moreton events, since weak disturbances, which are probably initiated more frequently, would not show up in $\mathrm{H} \alpha$. The apparent "velocity discrepancy" is further increased by this effect.

\subsection{Type II bursts as a signature of a common shock}

In all 12 events, metric type II radio bursts were present, which is another strong indication for the presence of an MHD shock 

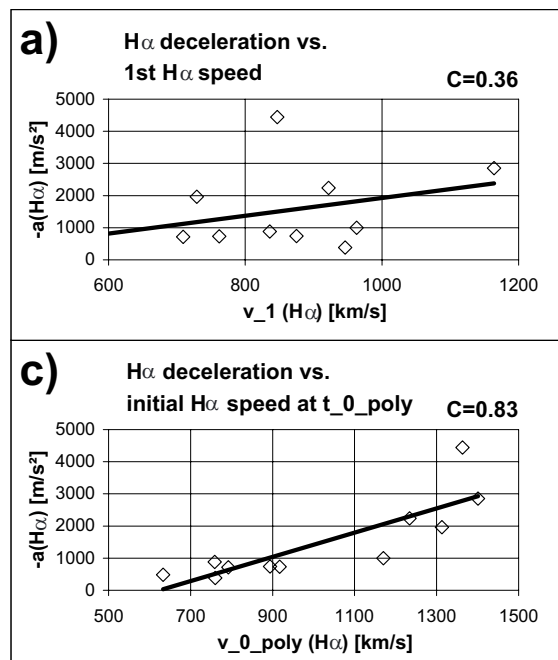

e) $\quad H \propto$ time delay (poly) vs.

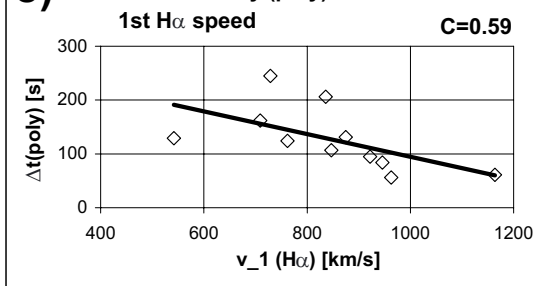

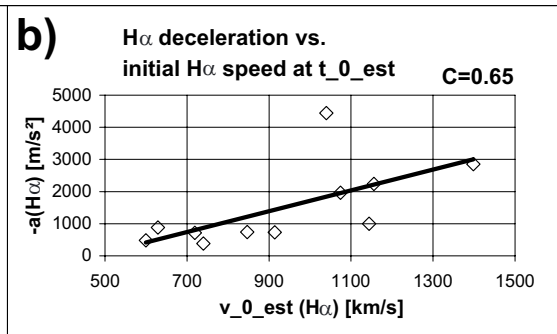

d) combined deceleration vs.

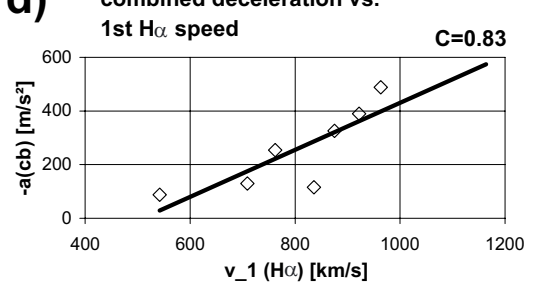

f) $\quad \mathrm{H} \alpha$ time delay (pl) vs.

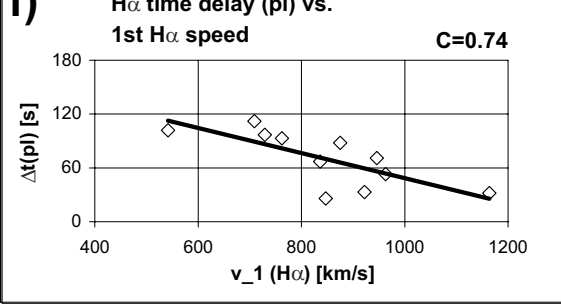

Fig. 5. a)-d): Flare wave accelerations $\left(\bar{a}_{\mathrm{H} \alpha}\right.$ and $\bar{a}_{c b}$ ) plotted versus various Moreton wave velocities. For convenience, the absolute values of $a$ are used. e), f): Moreton wave onset time delays $\Delta t$ (with respect to $t_{0 \text { poly }}$ and $t_{0 \mathrm{pl}}$ ) plotted versus the first measured Moreton wave velocity $v_{1}$. Linear fits of the data points are shown, and the correlation coefficients $C$ are indicated. in flare wave events. In two cases (E3 and E5), radioheliographic observations show a close temporal and spatial association between the bursts and the associated flare waves. In order to determine if the two phenomena are really created by the same shock, their kinematical properties must be compared. This is complicated by the dependence on coronal density models, which does not allow straightforward comparisons of the type II velocity and deceleration to the corresponding parameters of flare wave in terms of absolute values. However, several correlations between the kinematical parameters of type II bursts and Moreton waves can be established. They are presented in Fig. 6.

The velocities of the bursts and the Moreton waves are correlated, meaning that larger wave velocities also imply larger type II speeds. For example, the initial measured velocities $v_{1 \mathrm{H} \alpha}$ and $v_{\mathrm{b} \text { II }}$ yield a correlation coefficient of $C=0.88$ (see Fig. 6a). While these statistics must be interpreted with caution due to the low number of data points, they do nevertheless show at least qualitatively that type II bursts and Moreton waves are closely associated and can be interpreted as signatures of a common disturbance.

Further important evidence that supports this scenario is the good correlation $(C=0.86)$ found between the time lags $\Delta t$ of the onset of the radio bursts and the Moreton waves (Fig. 6c) with respect to the beginning of the SXR flare ( $\left.t_{\mathrm{b} S X R}\right)$. The later the Moreton wave is observed, the later the burst will start, too, which implies that the expansion of the disturbance (i.e. the shock geometry) is broadly self-similar in the different events. Note that this correlation is the most significant one due to the larger number of data points, and it does not depend on any density model. The self-similar expansion is supported by the correlation (albeit weak at $C=0.53$ ) that was found between the initial observed Moreton distances $r_{\mathrm{H} \alpha}^{\mathrm{min}}$ and the starting frequencies $f_{\mathrm{b}}$ (Fig. 6d), which means that the type II bursts are starting at lower heights when the Moreton waves are starting closer to the source point.

Like Moreton waves, faster type II bursts have a tendency to be associated with stronger deceleration (Fig. 6e), which is yet further evidence for a decaying shock. There is also a slight trend ( $C=0.57$, see Fig. $6 \mathrm{f}$ ) that faster bursts start at higher frequencies, i.e. lower heights. Since $v_{\mathrm{A}}$ and $v_{\mathrm{ms}}$ decrease with height at low heights above an AR (Gopalswamy et al. 2001; Mann et al. 2003), a fast disturbance will reach the critical Mach number at a lower height and therefore type II bursts will be observed at higher frequencies.

All type II bursts started within 3 min of the first observation of an $\mathrm{H} \alpha$ wavefront. In seven out of the 11 events where the type II starting times could be determined, the bursts started after the first Moreton wavefront became visible, the other four bursts starting before the observed wave onset. The average time difference between the type II appearance and the first Moreton front, $t_{\mathrm{bII}}-t_{1 M}$, was $0.8 \pm 1.9 \mathrm{~min}$. This variation in behavior could be caused by the $3-\mathrm{D}$ distribution of $v_{\mathrm{ms}}$ within the AR. However, we note that in two of the cases where the type II started before the wave became visible (E3 and E9), the time difference was less than $20 \mathrm{~s}$, which is considerably less than the $\mathrm{H} \alpha$ image cadence. In addition, the exact determination of $t_{\mathrm{b} \text { II }}$ is difficult due to interference with other radio features; therefore it is quite possible that in E3 and E9 the bursts started quasi-simultaneously with the wavefronts. The other two events with earlier type II bursts (E7 and E8) were both located at the limb, which makes the detection of the wavefronts difficult. 


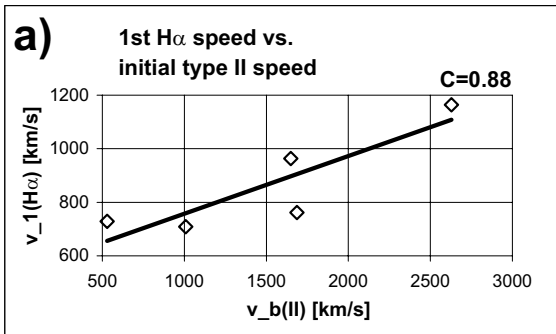

C) H $\alpha$ vs. type II
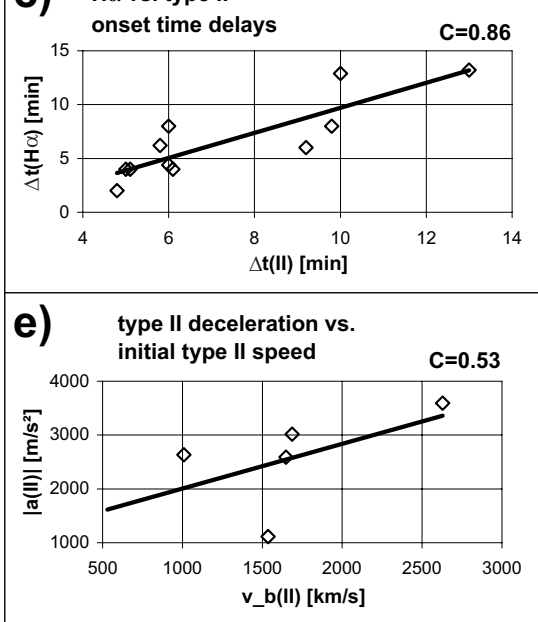

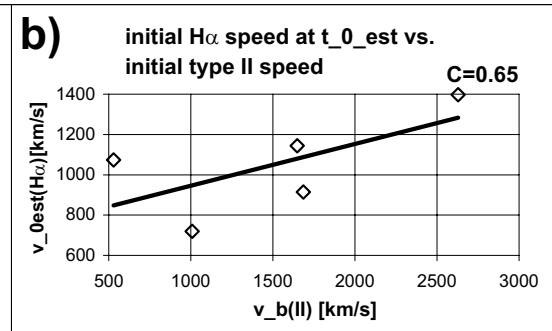

d) 1 st $\mathrm{H} \alpha$ distance vs.

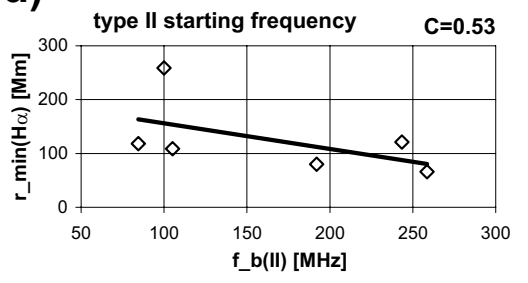

f)

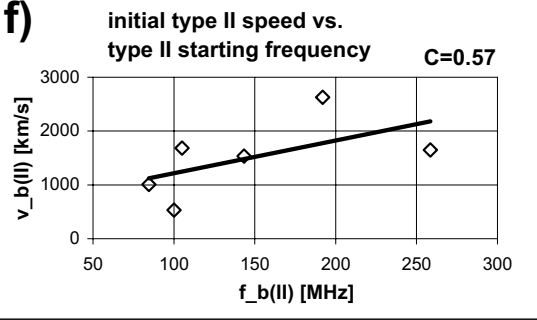

Fig. 6. a)-d): Correlations between parameters of type II bursts and Moreton waves. e), f): Correlations between different type II parameters. Linear fits of the data points are shown, and the correlation coefficients $C$ are indicated.
We conclude that at least in the majority of cases the type II burst starts slightly after the formation of the observable Moreton wave. This signifies that the perturbation usually needs more time to steepen into a shock in the vertical direction. This might be due to the larger $v_{\mathrm{ms}}$ in the higher corona (Mann et al. 1999). Note also that a disturbance propagating at $1000 \mathrm{~km} \mathrm{~s}^{-1}$ will cover a distance of $72 \mathrm{Mm}$ in $1.2 \mathrm{~min}$ (this is the average of $t_{\mathrm{b} I I}-t_{1 M}$ when we impose that the bursts which were observed before the first wavefronts had actually started simultaneously). Added to the average minimum Moreton distance $\left(\left\langle r_{\mathrm{H} \alpha}^{\min }\right\rangle=97 \pm 26 \mathrm{Mm}\right.$; see Paper I, Sect. 5.1), this gives $169 \mathrm{Mm}$, which is consistent with the average type II starting height of $\left\langle r_{\mathrm{b}}\right\rangle=181 \mathrm{Mm}$ deduced from the starting frequencies.

\subsection{The generation of flare wave signatures}

A schematic presentation of the shock is presented in Fig. 7, which is an expanded version of the scenario proposed by Vršnak et al. (2002a). Below the cartoon of the shock scenario we have included idealized plots of the intensity profiles $\Delta I_{\mathrm{c}}(r)$ for $\mathrm{H} \alpha_{\mathrm{c}}$ (solid line) and He I (dashed line), of the Doppler velocity $v_{\mathrm{D}}(r)$, and of the intensity profiles $\Delta I_{\mathrm{W}}(r)$ for the $\mathrm{H} \alpha$ line wings, $\mathrm{H} \alpha_{r}$ (solid) and $\mathrm{H} \alpha_{\mathrm{b}}$ (dashed).

Following the simulated wavefronts in Uchida et al. (1973) and $\mathrm{Wu}$ et al. (2001), the shock front is tilted towards the solar surface; this is caused by the increase of $v_{\mathrm{A}}$ with height in the low corona at larger distances from an AR (Mann et al. 1999). Such an inclined shock geometry is displayed by some EIT waves observed above the limb, as well as by the X-ray wave of 1998 May 6 (Hudson et al. 2003). The tilting is also consistent with the "premature" filament activation in E4 reported by Eto et al. (2002; see also Paper I, Sect. 6): the filament ( $\mathrm{F}$ in Fig. 7) is located higher up in the corona, and is therefore activated before the lower parts of the shock have actually reached it. On the disk, the visible EIT wavefront coincides with the lower part of the shock, since the largest fraction of the observed emission is generated there, and the comparatively tenuous upper parts of the wavefront are only observable in limb events.

The downstream coronal plasma is compressed and heated by the shock (shaded area in Fig. 7), which is observed as a front of increased emission by EIT and SXT. Higher up in the corona, the shock is able to accelerate electrons, which leads to radio emission observed as a metric type II burst. This unified explanation of flare waves and type II bursts was already proposed by Uchida (1974).

At the coronal base, the increased pressure behind the shock pushes down the chromospheric plasma (starting at $r=$ 2 in Fig. 7 and shown as small arrows in the $v_{\mathrm{D}}$ plot). During the downward motion (which has a velocity amplitude of 6-10 $\mathrm{km} \mathrm{s}^{-1}$, according to Švestka 1976), the Moreton wave is seen as a dark front in $\mathrm{H} \alpha_{r}$, and as a bright front in $\mathrm{H} \alpha_{\mathrm{b}}$, which is shown in the $\Delta I_{\mathrm{w}}$ plot in Fig. 7. After maximum compression is reached $(r=3)$, the chromosphere relaxes, creating the trailing disturbances in the $\mathrm{H} \alpha$ wings. Note that the chromosphere is comparatively inert and will not react instantaneously to the downstream pressure excess after the shock front passage. This smoothes out the leading edge of the Moreton wave perturbation profile and explains its non-shocklike shape.

If the excess emission in the $\mathrm{H} \alpha$ line center (shown in the $\Delta I_{\mathrm{c}}$ plot) was only due to the Doppler shift, the maximum of the intensity profile, $I_{\mathrm{m} H \mathrm{c}}$ (at $r=3$ ), would be reached when the downward motion stops, i.e. before the chromosphere starts 


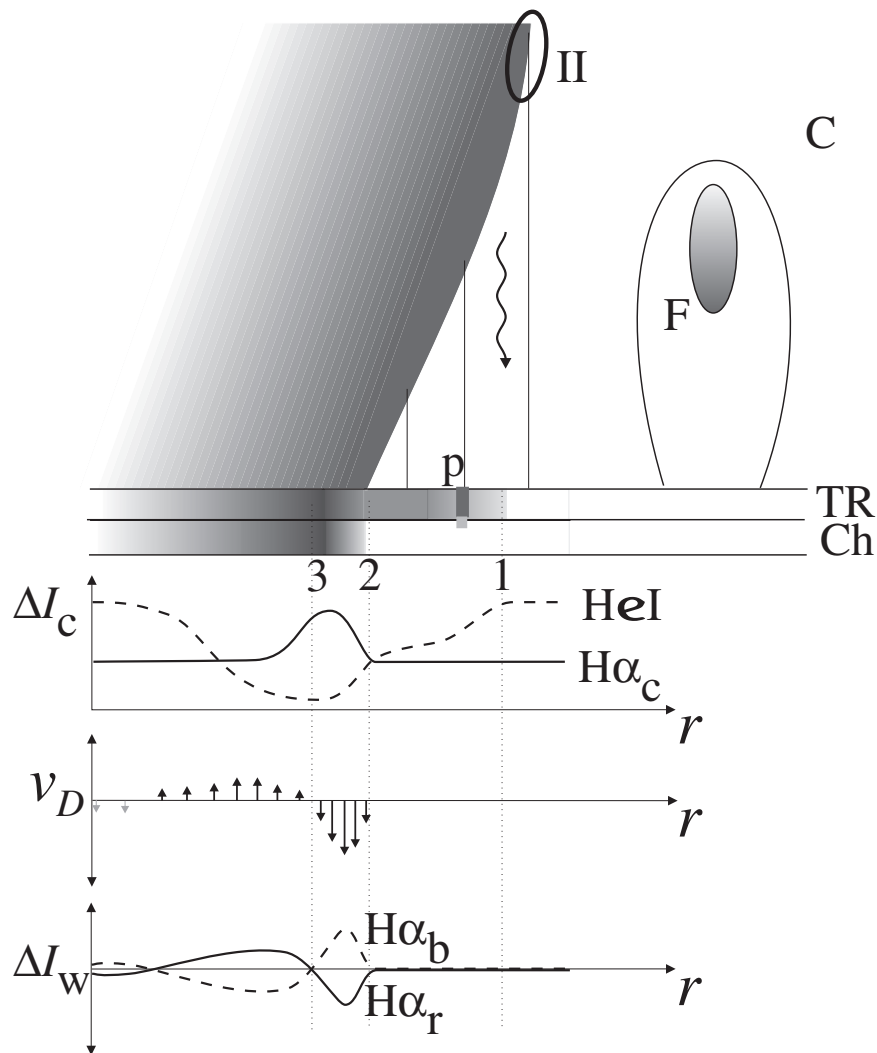

Fig. 7. Schematic presentation of the fast-mode MHD shock passage through the corona (C) and its signatures in the transition region (TR) and chromosphere $(\mathrm{Ch})$. For details see main text.

to "relax". Thus, $I_{\mathrm{m} H \mathrm{c}}$ should coincide with the maxima in the line wings. However, we found that it can be located anywhere between the line wing intensity maxima and the trailing edge of the wing wavefronts $(r=3)$. This variation is most probably caused by compressive heating of the chromosphere.

It is therefore reasonable to assume that both Doppler shifts and compressive heating contribute to the formation of the $\mathrm{H} \alpha$ line center signatures. Compression and/or heating is also revealed by the disturbances observed in the $17 \mathrm{GHz}$ radioheliograms, which showed a rise of the brightness temperature by up to $\approx 30 \%$.

It is noteworthy that the disturbances are never observed in the closest vicinity of the flare or the extrapolated starting point. For instance, the mean distance of the first observed $\mathrm{H} \alpha$ fronts from the calculated source point was $\left\langle r_{\mathrm{H} \alpha}^{\min }\right\rangle=97 \pm 26 \mathrm{Mm}$. Even at the estimated starting time $t_{0 \text { est }}$ the waves were not near the source: the extrapolated wavefront distance (using a 2nd degree polynomial fit) was still $\left\langle r_{0}\right\rangle=49 \pm 30 \mathrm{Mm}$ (see Paper I, Sect. 5.1). This can be explained by the time/distance the initial perturbation profile of the blast needs to steepen into a discontinuity (Vršnak \& Lulić 2000a). Before the shock formation the chromospheric plasma adjusts to a gradual coronal pressure increase through a series of quasi-equilibrium states, creating no $\mathrm{H} \alpha$ signatures. On the other hand, the observation that sometimes the $\mathrm{H} \alpha$ perturbation amplitude increases during the early propagation (see Sect. 3.1) may imply that the shock formation need not necessarily be completed when the disturbance becomes observable.

The passage of the shock results in an increase in density and temperature in the transition region as well. The He I absorption (dashed line in the $\Delta I_{\mathrm{c}}$ plot in Fig. 7) is enhanced due to an increase of collisional processes (see Vršnak et al. 2002a). This signature - its leading edge at $r=2$ is cospatial with the $\mathrm{H} \alpha$ wave - is the "main He I perturbation segment".

The more shallow He I "forerunner" (starting at $r=1$ ), on the other hand, must have a different origin. The PR mechanism (standing for photoionization-recombination; see Paper I, Sect. 2.2, and Vršnak et al. 2002a), which is enhanced by increased UV flux from the shocked coronal plasma, can account for the diffuse component of the disturbance, but not for its patchy appearance. Instead, the strong absorption increase in the He I mottles ("p" in Fig. 7) suggests that the magnetic field plays an important role in the formation of the forerunner. Since the shock is inclined, higher parts of the shock intersect magnetic field lines (thin vertical lines in Fig. 7) that are connected to surface areas ahead of the shock at the coronal base. This would allow some "signal" to propagate downwards along the field lines and create the forerunner.

In Vršnak et al. (2002a), two possibilities for such a signal were proposed: a thermal conduction front, which would enhance collisional processes in the transition region ahead of the intersection point ( $2>r>1$ in Fig. 7), and fast electrons, which are accelerated at the quasi-perpendicular section of the shock (observed as "herringbones" in type II radio bursts; see, e.g., Stewart \& Magun 1980) and which could then collisionally increase the He I absorption. The latter mechanism could possibly lead to fast-drifting microwave bursts during the time of the type II burst (cf. Karlický \& Odstrčil 1994).

The signal which propagates down along the magnetic field lines might also be able to explain the compact brightenings seen in $\mathrm{H} \alpha$ (see Paper I, Sect. 3.1), which are also sometimes located ahead of the Moreton fronts. However, the correspondence with the photospheric magnetic field is poorer than in the case of $\mathrm{He}$ I (only some $50 \%$ of the $\mathrm{H} \alpha$ brightenings are clearly associated with magnetic field concentrations).

Finally, let us briefly discuss coronal dimming, which was observed in all flare wave events with EIT data coverage and which was probably also detected in the He I data (see Sects. 3.2 and 3.3 in Paper I; Vršnak et al. 2002a). Dimming is commonly interpreted as a depletion of coronal material (e.g. Gopalswamy \& Thompson 2000; Khan \& Hudson 2000; Harrison et al. 2003). Since nearly all events were also associated with a CME, the dimming could be caused by the expansion of the erupting volume and its interaction with the ambient magnetic fields, and may not be related to the flare wave itself. In some recent models (Delannée 2000; Chen et al. 2002), the CMEs themselves are identified as the driver of the waves, so in these views the term "flare wave" would actually be a misnomer. We will address the question of the physical cause of the waves in a forthcoming paper, but our preliminary results indicate that flares still remain of central importance for the wave generation. 


\section{Conclusion}

A multiwavelength study of 12 flare wave events has been conducted. All events were observed in $\mathrm{H} \alpha$, and additional wave signatures were also detected in EIT Fe XII images, in He I filtergrams, in Yohkoh/SXT images, and in $17 \mathrm{GHz}$ radioheliograms. Let us once again summarize the most important observational findings:

- Flare waves can be observed in $\mathrm{H} \alpha$ (line center and wings), EUV, He I, SXR, and in microwaves. A disturbance of sufficient amplitude will create wave signatures in all these bands.

- In most events, the leading edge of the wavefronts initially has a nearly perfect circular curvature. This is not only the case for $\mathrm{H} \alpha$ waves, but also for EIT and He I fronts that are comparatively close to the source point.

- The extrapolated source points of the waves are clearly displaced from the central parts of the flare.

- The wavefronts in the different spectral channels are nearly cospatial, with the exception of the He I forerunner.

- All flare waves in our sample are decelerating, with the rate of deceleration becoming lower with increasing time/distance.

- The wavefronts in the different spectral channels follow closely associated kinematical curves.

- The intensity profiles show perturbation thickness increase and amplitude decrease with increasing time/distance.

- The waves are compressive, which is revealed by the emission measure increase observed in the EUV, SXR and microwave regimes, as well as by the downward swing of the chromosphere that was observed using $\mathrm{H} \alpha$ line wing data.

- All flare waves in our sample are associated with metric type II radio bursts.

- The type II bursts are more energetic and start at lower altitudes than those in a typical type II sample.

- The Moreton waves and type II bursts appear roughly at the same time. The onset times are correlated in the sense that the earlier the Moreton wave is first observed (with respect to the flare onset), the earlier the type II burst will start, too.

- Various kinematical parameters of type II bursts and Moreton waves are correlated.

- The deceleration rates and onset time delays of Moreton waves are correlated with their velocities.

The fact that the flare waves seen in the different spectral channels all follow closely associated kinematical curves implies that they are caused by a common physical disturbance. Furthermore, the waves do not propagate with constant velocities; instead, all flare waves are decelerating. The deceleration rate becomes smaller with increasing distance from their source point.

It was shown that there is no "velocity discrepancy" between Moreton and EIT waves. The differences in the measured speeds can be explained by the deceleration of the disturbance, combined with the fact that its signatures can be traced to much larger distances in EIT than in $\mathrm{H} \alpha$, and with the low image cadence of EIT.
The nearly circular curvature of the leading edges of the earliest wavefronts suggests that the underlying disturbance has a wave-like nature. On the other hand, the analysis of the intensity profiles of the flare waves has revealed that the broadening of the intensity profile and the decrease of its amplitude with increasing distance and time are the basic characteristics of the perturbation that causes flare waves. This behavior, combined with the deceleration of the disturbance, is expected for a freely propagating fast-mode MHD shock created by a large-amplitude perturbation (a nonlinear "simple wave"). As the shock propagates, its amplitude decreases, which also leads to a deceleration of the disturbance. Finally, the shock may decay to an ordinary fast-mode MHD wave.

This scenario is supported by the propagation speeds of the flare waves: the initial observed velocities of the $\mathrm{H} \alpha$ waves is $\left\langle v_{1 \mathrm{H} \alpha}\right\rangle=845 \mathrm{~km} \mathrm{~s}^{-1}$, while the velocities in the final part of the propagation of the disturbances, as indicated by measurements of the EIT waves, are $\left\langle\bar{v}_{\text {EIT }}\right\rangle=311 \mathrm{~km} \mathrm{~s}^{-1}$. Since the magnetosonic speed in the quiet low corona is, approximately, $v_{\mathrm{ms}}=200-600 \mathrm{~km} \mathrm{~s}^{-1}$, this means that initially the perturbations are clearly supermagnetosonic, with Mach numbers of $M_{\mathrm{ms}} \approx 1.5-4$, while their velocity towards the end of the observable propagation has dropped to about the magnetosonic speed $\left(M_{\mathrm{ms}} \approx 1\right)$. This behavior would be expected of a shock that gradually decays to an ordinary fast-mode wave.

The presence of a shock is further underlined by the observation of metric type II radio bursts in all Moreton events. The kinematics of the bursts are correlated with the kinematics of the Moreton waves (the same is true for the onset times), implying that both phenomena are signatures of the same shock. In two events, radioheliographic observations in the metric range have revealed that the locations of the type II burst sources are associated with the wavefronts (Pohjolainen et al. 2001; Khan \& Aurass 2002), and that their motions are generally consistent with the propagation of the flare waves.

These results clearly show that the "classical" fast-mode shock scenario is supported by recent multiwavelength observations. Alternative proposals, such as the CME-related scenarios of Delannée (2000) and Chen et al. (2002), are not able to explain the behavior of the flare waves in our sample adequately (see also Paper I, Sect. 6). However, we once again stress that we have studied only events with prominent $\mathrm{H} \alpha$ wave signatures, which probably represents a distinct class of disturbances - namely those associated with a coronal fastmode MHD shock. We do not rule out the possibility that a certain percentage of propagating disturbances, such as EIT waves that are not associated with Moreton signatures, are created by other mechanisms.

Some observational results still require explanation. For example, the fact that - despite large efforts - only $12 \mathrm{H} \alpha$ Moreton waves were identified implies that they are a comparatively rare phenomenon. Their occurrence rate is roughly one order of magnitude lower than that of both EIT waves and type II bursts, yet they were found to be closely associated with the latter two phenomena. This question is closely related with the main issue that has to be resolved: the initiation mechanism of the shock. There are several possible candidates, e.g. flare-generated pressure pulses, flare ejecta 
and CMEs. We will address these questions in forthcoming papers using the presented data set.

Acknowledgements. The work of A.W. was supported by DLR under grant No. 50 QL 0001. H $\alpha$ data are used with permission of BBSO, New Jersey Institute of Technology, and we thank the BBSO staff for their kind support. We are very grateful to H. Kurokawa and K. Shibata for the provision of $\mathrm{H} \alpha$ data from Hida Observatory. He I data were provided by the High Altitude Observatory of the National Center for Atmospheric Research, which is sponsored by the National Science Foundation, and we thank T. Holzer, H. Gilbert and A. Stanger for their support. Radioheliograms from the Nobeyama Radio Observatory were kindly provided by K. Shibasaki. Radiospectra were used with permission of IPS Radio and Space Services, Culgoora Solar Observatory, Narrabi, Australia, and we thank CRL (Japan) for providing on-line HiRAS data. We thank B. J. Thompson for the provision of $\mathrm{H} \alpha$ data, A. Vourlidas for corrected EIT times, and G. Mann, H. Aurass, and A. Klassen for helpful discussions. $\mathrm{SOHO}$ is a project of international cooperation between ESA and NASA. Yohkoh is a mission of the Institute of Space and Astronautical Sciences (Japan), with participation from the US (NASA) and UK (PPARC). A.W. and A.H. acknowledge the support of the Austrian FWF Project P13653-PHY.

\section{References}

Chen, P. F., Wu, S. T., Shibata, K., \& Fang, C. 2002, ApJ, 572, L99 Delaboudinière, J.-P., Artzner, G. E., Brunaud, J., et al. 1995, Sol. Phys., 162, 291

Delannée, C. 2000, ApJ, 545, 512

Denker, C., Johannesson, A., Marquette, W., et al. 1999, Sol. Phys., 184,87

Eto, S., Isobe, H., Narukage, N., et al. 2002, PASJ, 54, 481

Gopalswamy, N., \& Thompson, B. J. 2000, J. Atmos. and Sol.-Terr. Phys., 62, 1457

Gopalswamy, N., Lara, A., Kaiser, M. L., \& Bougeret, J.-L. 2001, J. Geophys. Res., 106, 25261

Harra, L. K., \& Sterling, A. C. 2003, ApJ, 587, 429

Harrison, R. A., Bryans, P., Simnett, G. M., \& Lyons, M. 2003, A\&A, 400, 1071

Harvey, K. L., Martin, S. F., \& Riddle, A. C. 1974, Sol. Phys., 36, 151

Hudson, H. S., Khan, J. I., Lemen, J. R., Nitta, N. V., \& Uchida, Y. 2003, Sol. Phys., 212, 121

Karlický, M., \& Odstrčil, D. 1994, Sol. Phys., 155, 171

Kerdraon, A., \& Delouis, J.-M. 1997, in Coronal Physics from Radio and Space Observations, ed. G. Trottet (Berlin: Springer), Lect. Notes Phys., 483, 192

Khan, J. I., \& Hudson, H. S. 2000, GRL, 27, 1083

Khan, J. I., \& Aurass, H. 2002, A\&A, 383, 1018

Klassen, A., Aurass, H., Klein, K.-L., Hofmann, A., \& Mann, G. 1999, A\&A, 343, 287

Klassen, A., Aurass, H., Mann, G., \& Thompson, B. J. 2000, A\&AS, 141,357

Klein, K.-L., Khan, J. I., Vilmer, N., Delouis, J.-M., \& Aurass, H. 1999, A\&A, 346, L53

Kondo, T., Isobe, T., Igi, S.,Watari, S., \& Tokumaru, M. 1995, J. Commun. Res. Lab., 42, 111

Kurokawa, H., Ishiura, K., Kimura, G., et al. 1995, J. Geomag Geoelecter, 47, 1043
Landau, L. D., \& Lifshitz, E. M. 1987, Fluid Mechanics, 2nd ed. (Oxford: Pergamon)

MacQueen, R. M., Blankner, J. G., Elmore, D. F., Lecinski, A. R., \& White, O. R. 1998, Sol. Phys., 182, 97

Mann, G., Aurass, H., Voigt, W., \& Paschke, J. 1992, in Proc. 1st SOHO Workshop, ESA SP-348, 129

Mann, G. 1995, J. Plasma Phys., 53, 109

Mann, G., Aurass, H., Klassen, A., Estel, C., \& Thompson, B. J. 1999, in Proc. 8th SOHO Workshop, ed. B. Kaldeich-Schürmann, ESA SP-446, 477

Mann, G., Klassen, A., Aurass, H., \& Classen, H.-T. 2003, A\&A, 400, 329

Messerotti, M., Otruba, W., Warmuth, A., et al. 1999, in Proc. ESA Workshop on Space Weather, ed. N. Crosby, ESA WPP-155, 321

Moreton, G. E., \& Ramsey, H. E. 1960, PASP, 72, 357

Murawski, K., Nakariakov, V. M., \& Pelinovsky, E. N. 2001, A\&A, 366, 306

Nakajima, H., Nishio, M., Enome, S., et al. 1994, Proc. IEEE, 82, 705

Narukage, N., Hudson, H. S., Morimoto, T., et al. 2002, ApJ, 572, L109

Nelson, G. S., \& Melrose, D. B. 1985, in Solar Radiophysics, ed. D. J. McLean, \& N. R. Labrum (Cambridge: Cambridge Univ. Press), 333

Newkirk, G. A. 1961, ApJ, 133, 983

Ofman, L., \& Thompson, B. J. 2002, ApJ, 574, 440

Pohjolainen, S., Maia, D., Pick, M., et al. 2001, ApJ, 556, 421

Prestage, N. P., Luckhurst, R. G., Paterson, B. R., Bevins, C. S., \& Yuile, C. G. 1994, Sol. Phys., 150, 393

Priest, E. R. 1982, Solar Magnetohydrodynamics (Dordrecht: Reidel)

Robinson, R. D. 1985, Sol. Phys., 95, 343

Saito, K. 1970, Ann. Tokyo Astr. Obs., 12, 53

Scherrer, P. H., Bogart, R. S., Bush, R. I., et al. 1995, Sol. Phys., 162, 129

Shibata, K., Eto, S., Narukage, N., et al. 2002, in Proc. Yohkoh 10th Anniversary Meeting, ed. P. Martens, Cospar Colloq., 13, 279

Stewart, R. T., \& Magun, A. 1980, Proc. ASA, 4, 53

Švestka, Z. 1976, Solar Flares (Dordrecht: Reidel)

Thompson, B. J., Plunkett, S. P., Gurman, J. B., et al. 1998, Geophys. Res. Lett., 25, 2465

Tsuneta, S., Acton, L., Bruner, M., et al. 1991, Sol. Phys., 136, 37

Uchida, Y. 1968, Sol. Phys., 4, 30

Uchida, Y., Altschuler, M. D., \& Newkirk, G., Jr. 1973, Sol. Phys., 28, 495

Uchida, Y. 1974, Sol. Phys., 39, 431

Vršnak, B., \& Lulić., S. 2000a, Sol. Phys., 196, 157

Vršnak, B., \& Lulić., S. 2000b, Sol. Phys., 196, 181

Vršnak, B., Magdalenić, J., \& Aurass, H. 2001, Sol. Phys., 202, 319

Vršnak, B., Warmuth, A., Brajša, R., \& Hanslmeier, A. 2002a, A\&A, 394, 299

Vršnak, B., Magdalenić, J., Aurass, H., \& Mann, G. 2002b, A\&A, 396, 673

Wang, Y.-M. 2000, ApJ, 543, L89

Warmuth, A., Vršnak, B., Aurass, H., \& Hanslmeier, A. 2001, ApJ, 560, L105

Warmuth, A., Vršnak, B., Magdalenić, J., Hanslmeier, A., \& Otruba, W. 2004, A\&A, 418, 1101

Wu, S. T., Zheng, H., Wang, S., et al. 2001, J. Geophys. Res., 106, 25089 